\section{GRP-082 HOSPITAL PHARMACIST INTERVENTIONS IN PATIENTS WITH ENTERAL NUTRITION FEEDING TUBES}

doi:10.1136/ejhpharm-2013-000276.082

'P López Sánchez, 'MC Conde García, ${ }^{2 T}$ Sánchez Casanueva, 'S Canales Ugarte, 'E Zamora Ferrer, 'JC Valenzuela Gámez. 'H.G. La Mancha Centro, Pharmacy Service, Alcázar de San Juan, Spain; ${ }^{2}$.G. Tomelloso, Pharmacy Service, Tomelloso, Spain

Background Patients receiving enteral nutrition (EN) suffer several kinds of complications such as gastrointestinal disorders, lung aspiration, tube dislodgement, tube clogging, hyperglycaemia and electrolytic alterations. The pharmacist's key role is to ensure the best nutrition and to solve and prevent problems related to drug administration by this route.

Purpose To analyse hospital pharmacist interventions (HPIs) in patients fed with EN through feeding tubes.

Materials and Methods Prospective study from 1 July 2011 to 30 June 2012 in a 350-bed hospital. Twice a week a hospital pharmacist monitored patients fed through feeding tubes. HPIs were classified in four types: Type 1: EN formula recommendation (to increase nutritional support, to recommend another kind of formula, to modify the regimen); Type 2: to recommend flushing feeding tubes with water; Type 3: to suggest replacing PVC nasogastric tubes (NTs) with silicone NTs; Type 4: to adjust pharmacotherapy (ENdrug interactions and drug incompatibilities). The pharmacist reported all HPIs to physicians.

Results A total of 132 patients were monitored, with 94 HPIs Type 1: 45 (47.9\%) (37 (82.3\%) to increase nutritional input, $2(4.4 \%)$ regimen modification and $6(13.3 \%)$ new formula recommendations), Type 2: 11 (11.7\%); Type 3: 3 (3.2\%); Type 4: $35(37.2 \%)$ (12 (34.3\%) substitutions of omeprazole capsules for omeprazole oral solution, 10 (28.6\%) lactulose for lactitol, $9(25.7 \%)$ delayed-release tablets for immediate-release tablets, 4 (11.4\%) others).

Conclusions HPIs contributed to improved pharmacotherapy and suitability of the EN formula in most of the patients with feeding tubes. Designing an EN multidisciplinary care plan improves patients' treatment and health outcomes.

No conflict of interest.

\section{GRP-083 HOSPITAL PHARMACISTS CAN IMPROVE PHARMACOVIGILANCE IN THE EMERGENCY ROOM}

doi:10.1136/ejhpharm-2013-000276.083

FA Aliberti, N Ciociano, L Grisi, MG Elberti, M Alfieri, GM Lombardi, F Romano. University Hospital "San Giovanni di Dio e Ruggi d'aragona" Salerno, Department of Pharmacy, Salerno, Italy

Background Hospital pharmacists can play an important role in reporting adverse drug reaction (ADRs). Several publications underscore the fact that adverse drug events account for a substantial percentage of all hospital admissions. In the literature, several ways are mentioned in which the pharmacist can contribute to the safe use of drugs.

Purpose To establish ADRs in the Emergency Room (ER).

Materials and Methods This study was conducted from April 2010 to December 2011 in Salerno University Hospital. ADR report forms completed in the first 20 months of the project were analysed. Some of their key principles were collected: sex; suspected drug that caused the reaction and other drugs taken in association; description of $\mathrm{ADRs}$ and their classification as non-severe, severe or life-threatening. They were compared with ADR data for 2009.

Results 158 forms were analysed, each related to one different patient: 98 patients were women (68\%). $50 \%$ of the events were connected with antibiotics, e.g. amoxicillin/clavulanic acid (28 cases), penicillin (19 cases), cephalosporins (17 cases); 35\% concerned antiinflammatories such as nimesulide (21\%), propionic acid derivatives (21\%), acetylsalicylic acid (14\%), ketorolac (11\%), steroidal antiinflammatories (7\%). 103 patients didn't take other drugs, but 55 had taken another one. Skin reactions were $52 \%$ of events, while $14 \%$ were cardiovascular events, $13 \%$ gastrointestinal problems, and $8 \%$ were respiratory reactions. Non-severe ADRs were $75 \% ; 25 \%$ were severe and 1 case life-threatening. Before the project, in 2009 only one ADR had been reported; zero reports in the period JanuaryMarch 2010.

Conclusions It is evident that the presence of a hospital pharmacist in ER increases the number of ADR reports: data confirms that a pharmacist who supports medical staff in reporting ADRs should be operative in all hospital departments.

No conflict of interest.

\section{GRP-084 HOW HAS THE INTRODUCTION OF NEW DRUG CHARTS AFFECTED PRESCRIBING DOCUMENTATION?}

doi:10.1136/ejhpharm-2013-000276.084

${ }^{1} \mathrm{H}$ Badham, ${ }^{2} \mathrm{~K}$ Westacott, ${ }^{2} \mathrm{D}$ Petrikova, ${ }^{2} \mathrm{~S}$ Dolling. 'University Hospitals Bristol, Pharmacy, Bristol, UK; '2University Hospitals Bristol, Medicine, Bristol, UK

Background University Hospitals Bristol (UHBristol) have standards for safe and professional prescribing [1]. The standards include prescriber accountability and informed clinical decision making by awareness of drug chart(s) in use and any medicine(s) not given. In 2011 the Medical, Pharmaceutical and Nursing Colleges produced standards for hospital in-patient prescription charts aimed to help eliminate prescribing errors and improve patient outcomes [2]. The standards correlate with the UHBristol standards.

Timeline Initial audit February 2010. New prescription chart was released in July 2010 and re-audited in September 2010. Revised chart was released July 2011 and re-audited in January 2012

Directing Change The audit results and the NHS Institute for Innovation and Improvement Plan, Do, Study, Act (PDSA) [3] tool informed each chart change. The strategy was co-ordinated by pharmacy, with input from the healthcare team.

Purpose To establish achievement of the prescribing standards below within in-patient medical wards at UHBristol.

Prescriber identity: $100 \%$ of prescribers should print their name

Prescriber contact: $100 \%$ of prescribers should print their bleep number

Additional chart(s): $100 \%$ of additional prescription charts(s) will be documented on main prescription chart

Missed doses: $100 \%$ of medicines that are not given will have a documented reason

Materials and Methods Data collection proforma was designed, piloted and used for each audit cycle. Ten in-patient prescription charts from each ward were reviewed.

Results The table states the achievement of the standards with each cycle. The last column indicates the change between the first and last audit.

Conclusions Each revision of the prescription chart produced improvements in achievement of the standards. The audit cycle, PDSA and multidisciplinary approach informed changes and enhanced the charts' fitness for purpose.

\section{References}

1. UHBristol Medicines Governance Group.Medicines code.2009.

2. http://www.rpharms.com/what-s-happening-/news_show. asp?id $=275$

3. http://www.ihi.org/knowledge/Pages/Tools/PlanDoStudy ActWorksheet.aspx 